\title{
Successful Resection of Lymphangioma of the Neck and Mediastinum Presenting as Stridor in a 12 Year Old Child
}

\author{
Babar Irfan ${ }^{1}$, Maha Tahir ${ }^{2}$, Omar Irfan ${ }^{3}$, Hamza Abdur Rahim Khan ${ }^{3}$, Saulat H. Fatimi ${ }^{3}$ \\ ${ }_{1}^{1}$ Jinnah Sindh Medical University, Karachi, Pakistan, ${ }^{2}$ Dow International Medical College, Karachi, \\ Pakistan, ${ }^{3}$ Aga Khan University Medical College, Karachi, Pakistan
}

\begin{abstract}
Keywords:
Lymphangioma, mediastinum, stridor, cervical mass.

Abstract:

Lymphangiomas are congenital malformations of lymphatic tissue. This benign condition is generally found in the neck region and is discovered by 2 years of age. Here we report the case of a 12 year old child who presented with a cervical and mediastinal cystic hygroma that had progressed to such a space occupying nature as to cause the shifting of the trachea. The entire mass was successfully resected with a lower neck incision. Our case suggests the necessity of considering cystic lymphangioma in the differential diagnosis of lateral neck masses and use of adequate imaging to substantiate the pre-operative diagnosis.
\end{abstract}

(Cardiovasc. j. 2017; 9(2): 167-169)

\section{Introduction:}

Lymphangiomas are congenital malformations of the lymphatic system. Usually they present at birth or develop later during infancy. $90 \%$ of cases however, are detected by 2 years of age. ${ }^{1}$ Lymphangiomas are typically located in the neck $(75 \%)$ and axilla (20\%) while only $3 \%-10 \%$ of neck lesions extend into the mediastinum and about $1 \%$ are present entirely in the mediastinum. ${ }^{2}$ They have a prevalence of less than $4.5 \%$ of all mediastinal tumors. ${ }^{3}$

The typical presentation is a soft, non-tender, smooth mass that is compressible and can be transilluminated; with alteration in size resulting from infection or hemorrhage. There may be respiratory compromise and dysphagia along with aesthetic anomaly depending upon the location of the mass, the bulk and the degree of compression on the surrounding structures. In the majority of the cases the child presents without any functional problems because the cosmetic appearance brings attention much earlier in the disease course.

Lymphangiomas are diagnosed based on clinical history, examination and imaging. Ultrasound and CT scans can help in the diagnosis of the disease. However, MRI remains the imaging modality of choice for a definitive diagnosis as well as for determining the intra-thoracic extension of the lesion. Diagnosis is confirmed by postoperative histology. ${ }^{4}$ Nevertheless, older children presenting with a neck swelling accompanied by symptoms of respiratory discomfort are likely to be misdiagnosed in favor of an infectious cause.

\section{Case Report:}

A 12 year old child presented to our hospital with two months history of shortness of breath that was progressively worsening and developed stridor. There were no other symptoms, no comorbid conditions, no family history of similar complaints and normal birth and development. Physical examination revealed a fluctuant large mass in the left half of the neck with deviation of the trachea onto the right side. CT scan of the neck was performed that showed a large lymphangioma in the neck and upper mediastinum with compression of the mid and lower trachea (Figure 1). Based on these findings, surgical resection of the mass was planned. Via a left lateral neck incision with extension up to supra sternal notch, the entire lymphangioma was resected successfully (Figure 2). Sternotomy was not required. Postoperative course was unremarkable and patient was discharged home in perfect health after four days. The patient was followed for another 6 months without any signs for post-operative complications or recurrence of the disease.

Address of Correspondence: Dr. Omar Irfan, Aga Khan University Medical College, Karachi, Pakistan. Email:omarirfan1@hotmail.com 

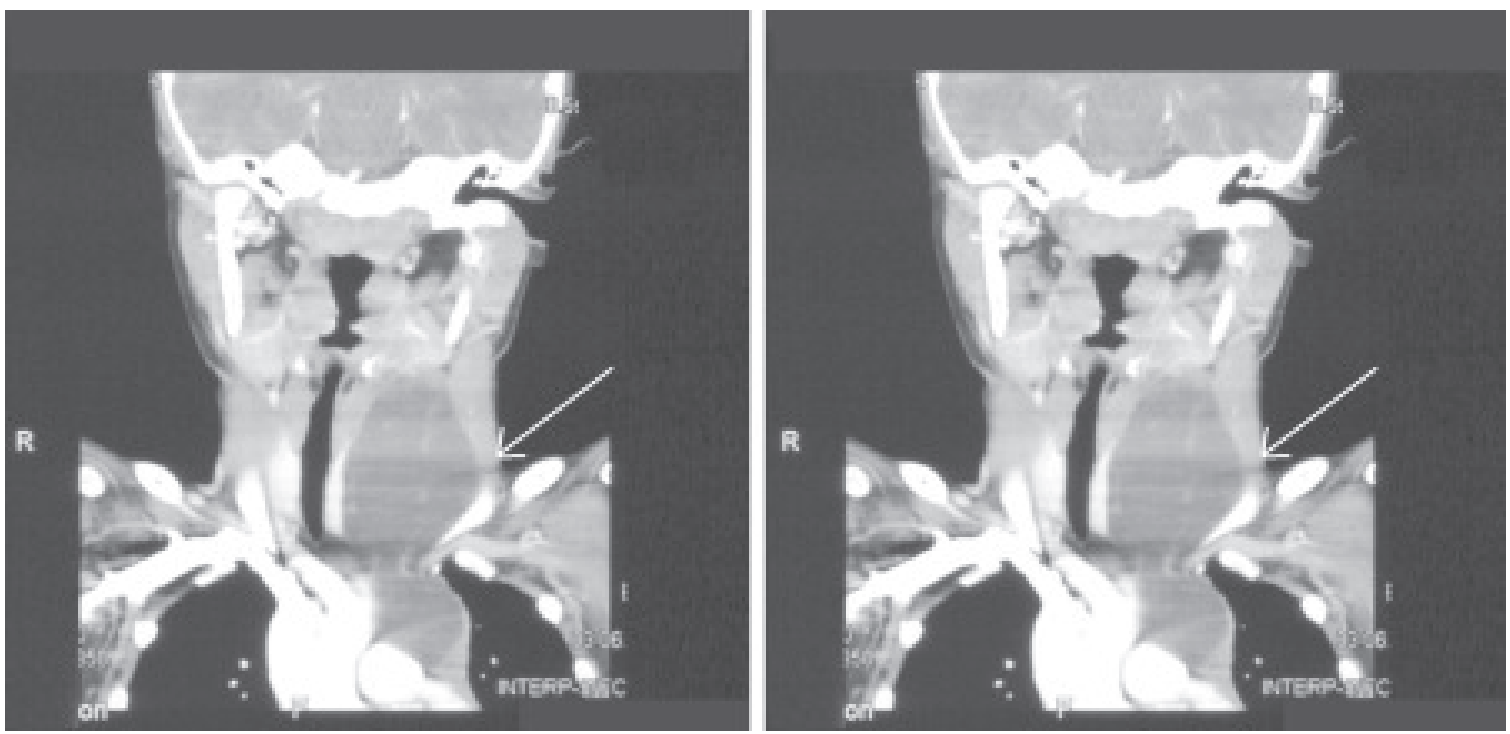

Fig.-1: $C T$ scan showing the lesion in the neck extending up to the aorticopulmonary window in the mediastinum (as indicated by the arrow). This mass was shifting the trachea to the right.

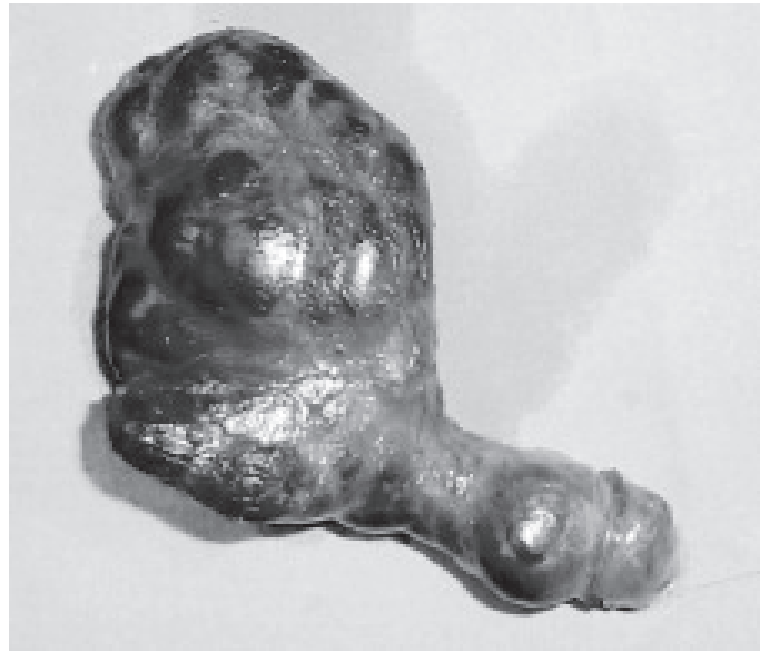

Fig.-2: Resected Lymphangioma.

\section{Discussion:}

Lymphangiomas are malformations of lymph tissue that results from the failure of lymph spaces to connect to the rest of the lymphatic system. Malformations that contain both lymphatic and venous components are called Venolymphatic malformations. Macrocystic lymphatic malformations (cystic hygroma) are lesions that contain large thick-walled cysts that have little infiltration of surrounding tissue allowing for a relatively straight-forward excision procedure. Microcystic lymphatic malformations are lesions with more extensive infiltration in the floor of mouth and tongue along with other soft-tissue structures of the head and neck, making their excision difficult.

Lymhangiomas can be classified according to two methods. Classification can be based on size, dividing the malformation into microcystic or capillary lymphangiomas, in which the lesions are less than $1 \mathrm{~cm}$ in diameter, and macrocystic cystic hygromas, in which the cysts are larger than 1 $\mathrm{cm} .{ }^{5}$ The other classification is based on location and extent of the involvement. De Serres and colleagues proposed a five-stage classification for lymphangiomas depending on whether the lesion was unilateral or bilateral and suprahyoid or infrahyoid. ${ }^{6}$

Surgical resection is the treatment of choice for lymphangiomas. The purpose of surgery is to improve cosmetic appearance, offset any difficulty in eating or breathing and to carry out a complete excision of the lesion(s) without causing damage to important neck structures and facial musculature. ${ }^{5}$ De Serres and colleagues evaluated postoperative complications of 56 patients who underwent surgical resection of cervical lymphangiomas. ${ }^{6}$ These complications included cranial nerve paralysis, infection, and seroma formation. Based on their proposed anatomic staging system for lymphangiomas, the percentage of complications ranged from $17 \%$ in stage I (unilateral infrahyoid) to $100 \%$ in stage V (bilateral 
supra- and infrahyoid). Long-term morbidity included cosmetic deformity, speech problems, and malocclusions and was more frequent in higher staged lesions. Surgical removal is usually more difficult in children than in adults, and lesions in children have higher recurrence rates after complete excision. ${ }^{7}$

Sclerotherapy of lymphangiomas is a recognized alternative treatment. Macrocystic lesions can be treated with sclerotherapy using alcohol. Baskota et al. reported total resolution in nine out of ten patients treated with a single intralesional injection of OK- $432 .{ }^{8}$ Several other agents have been used, such as sodium morrhuate, doxycycline, bleomycin, dextrose, tetracycline and ethanol. ${ }^{9}$ The main complication of this therapy is the possible diffusion of the sclerosing agent into the tissues surrounding the lymphangioma, which causes tissue fibrosis and complicates an eventual complementary surgery.

\section{Conclusion}

Through this report, we recommend the need to have cystic lymphangioma as an important differential diagnosis of neck masses in children even in areas like Pakistan where Tuberculosis is the number one suspect in lesions of a space occupying nature. Patients, who present with neck swellings, are treated according to the guidelines of Tuberculosis, especially those who cannot afford a complete workup. This leads to a delay in the actual diagnosis. This case was atypical in that a cervical cystic hygroma presented in a child as a mediastinal mass that was large enough to cause shifting of the trachea and required a thorough investigation. The entire mass was successfully resected with a lower neck incision and the patient is without signs of recurrence 6 months after surgery.

\section{Conflict of Interest - None.}

\section{References}

1. Giguere CM, Bauman NM, Smith RJ. New treatment options for lymphangioma in infants and children. Ann Otol Rhinol Laryngol 2002 Dec;111(12 Pt 1):1066-1075.

2. Kraus, J., et al., Cystic lymphangioma of the neck in adults: a report of three cases. Wien Klin ochenschr 2008. 120(7-8): 242-245.

3. Park, J.G., et al., Mediastinal lymphangioma: Mayo Clinic experience of 25 cases. Mayo Clin Proc 2006. 81(9): 1197-203

4. Munoz Herrera A, Perez Plasencia D, Gomez Benito M, Santa Cruz Ruiz S, Flores Corral T, Aguirre Garcia F. [Cervical lymphangioma in adults. Description of 2 cases]. Ann Otolaryngol Chir Cervicofac 1998 Nov;115(5):299-302.

5. Gross E, Sichel JY. Congenital neck lesions. Surg Clin North Am 2006 Apr;86(2):383-392, ix.

6. de Serres LM, Sie KC, Richardson MA. Lymphatic malformations of the head and neck. A proposal for staging. Arch Otolaryngol Head Neck Surg 1995 May;121(5):577-582.

7. Karkos PD, Spencer MG, Lee M, Hamid BN. Cervical cystic hygroma/lymphangioma: an acquired idiopathic late presentation. J Laryngol Otol 2005 Jul;119(7): 561-563.

8. Baskota DK, Singh BB, Sinha BK. OK-432: an effective sclerosing agent for the treatment of lymphangiomas of head and neck. Kathmandu Univ Med J (KUMJ) 2007 Jul-Sep;5(3):312-317.

9. Molitch HI, Unger EC, Witte CL, vanSonnenberg E. Percutaneous sclerotherapy of lymphangiomas. Radiology 1995 Feb;194(2):343-347. 